\title{
COMPLETION OF A GAUSSIAN DERIVATION
}

\author{
JOHN A. EWELL
}

ABstract. The author supplies evaluation of two coefficients of an identity partially derived by Gauss. Several special cases of this identity are then presented, the last of these identities yielding an alternate proof of Ramanujan's theorem concerning divisibility of certain values of the partition function by the modulus 7 .

1. Introduction. In this paper we investigate the following identity

$$
\begin{aligned}
& \prod_{n=1}^{\infty}\left(1+a x^{2 n-1}\right)^{2}\left(1+a^{-1} x^{2 n-1}\right)^{2} \\
& \quad=A_{0}(x) \sum_{m=-\infty}^{\infty} a^{2 m} x^{2 m^{2}}+A_{1}(x) \sum_{m=0}^{\infty} x^{2 m(m+1)}\left(a^{2 m+1}+a^{-2 m-1}\right)
\end{aligned}
$$

which according to MacMahon [3, p. 79] was first derived by Gauss. The derivation is valid for each pair of complex numbers $a, x$ such that $a \neq 0$ and $|x|<1$. It seems that the coefficients $A_{0}(x), A_{1}(x)$ were left undetermined, and apparently have never been determined. We here set for ourselves the goal of filling this gap. Specifically, in $\$ 2$ we prove

THEOREM 1. For each given pair of complex numbers $a$, $x$ with $a \neq 0$ and $|x|<1$,

$$
\begin{aligned}
& \prod_{n=1}^{\infty}\left(1-x^{2 n}\right)^{2}\left(1+a x^{2 n-1}\right)^{2}\left(1+a^{-1} x^{2 n-1}\right)^{2} \\
& \quad=\sum_{m=-\infty}^{\infty} x^{2 m^{2}} \sum_{m=-\infty}^{\infty} a^{2 m} x^{2 m^{2}} \\
& \quad+x \sum_{m=-\infty}^{\infty} x^{2 m(m+1)} \sum_{m=0}^{\infty} x^{2 m(m+1)}\left(a^{2 m+1}+a^{-2 m-1}\right) .
\end{aligned}
$$

$\$ 3$ is devoted to the derivation of four special cases of identity (1). The fourth of these identities expresses the infinite product $\Pi\left(1-x^{n}\right)^{6}$ as a sum of two double series. In $\$ 4$, we then sketch an argument which shows that this identity yields an alternate proof of Ramanujan's theorem concerning divisibility of certain values of the partition function by the modulus 7 .

2. Proof of Theorem 1. For given $a, x$ let the infinite product $G(a, x)$ be defined by

$$
G(a, x)=\prod_{n=1}^{\infty}\left(1+a x^{2 n-1}\right)^{2}\left(1+a^{-1} x^{2 n-1}\right)^{2}
$$

Received by the editors January 26, 1981 and, in revised form, May 22, 1981.

Key words and phrases. Identities, partition function, triple-product identity. 1980 Mathematics Subject Classification. Primary 05A19; Secondary 05A17. 
Then, for each pair of positive real numbers $A, X$, with $X<1, G(a, x)$ converges absolutely and uniformly for all pairs $a, x$ such that $A^{-1} \leqslant|a|<A$ and $|x|<X$. Hence, for a fixed choice of $x,|x|<1, G(a, x)$ defines a unique function of $a$, which is analytic at all points of the finite complex plane except $a=0$ where it has an essential singularity. Accordingly,

$$
G(a, x)=A_{0}(x)+\sum_{n=1}^{\infty}\left[A_{n}(x) a^{n}+A_{-n}(x) a^{-n}\right] .
$$

where the coefficients $A_{n}(x), A_{-n}(x)$ are uniquely determined by the chosen $x$.

Now, $G(a, x)=G\left(a^{-1}, x\right)$, whence $A_{n}(x)=A_{-n}(x)$, for each positive integer $n$. Hence,

$$
G(a, x)=A_{0}(x)+\sum_{n=1}^{\infty} A_{n}(x)\left(a^{n}+a^{-n}\right) .
$$

Next, we easily establish the identity $G\left(a x^{2}, x\right)=(a x)^{-2} G(a, x)$. With the help of (2) we expand both sides of this identity in powers of $a$, and subsequently equate coefficients of like powers to obtain the following recurrence: $A_{n+2}(x)=$ $A_{n}(x) x^{2(n+1)}$. We split cases according to the parity of $n$. In the case of $n$ even iteration of the recurrence yields the determination: $A_{2 m}(x)=A_{0}(x) x^{2 m^{2}}$, for each nonnegative integer $m$. For $n$ odd we have $A_{2 m+1}(x)=A_{1}(x) x^{2 m(m+1)}$, for each nonnegative integer $m$. Substituting these values into (2) we thus derive identity (0).

To evaluate the coefficients $A_{0}(x)$ and $A_{1}(x)$ we appeal to the well-known triple-product identity:

$$
\prod_{n=1}^{\infty}\left(1-x^{2 n}\right)\left(1+a x^{2 n-1}\right)\left(1+a^{-1} x^{2 n-1}\right)=\sum_{n=-\infty}^{\infty} a^{n} x^{n^{2}}
$$

For a proof see [2, p. 282]. We square both sides of the triple-product identity and determine the coefficients of $a^{0}$ and $a+a^{-1}$ for the right side of the resulting identity. Straightforward partial expansion yields

$$
\begin{aligned}
\left\{\sum_{n=-\infty}^{\infty} a^{n} x^{n^{2}}\right\}^{2}= & \sum_{m=-\infty}^{\infty} x^{2 m^{2}}+x \sum_{m=-\infty}^{\infty} x^{2 m(m+1)}\left(a+a^{-1}\right) \\
& + \text { a series in } a^{n}, a^{-n}, \quad n>1 .
\end{aligned}
$$

Between identity (0) and the square of the triple-product identity we eliminate the product $\Pi_{n=1}^{\infty}\left(1+a x^{2 n-1}\right)^{2}\left(1+a^{-1} x^{2 n-1}\right)^{2}$, and thereafter equate coefficients of $a^{0}$ and $a+a^{-1}$ to obtain

$$
\begin{aligned}
& A_{0}(x)=\prod_{n=1}^{\infty}\left(1-x^{2 n}\right)^{-2} \sum_{m=-\infty}^{\infty} x^{2 m^{2}}, \\
& A_{1}(x)=\prod_{n=1}^{\infty}\left(1-x^{2 n}\right)^{-2} x \sum_{m=-\infty}^{\infty} x^{2 m(m+1)} .
\end{aligned}
$$

Substituting these values into identity (0) we thus prove our theorem. 


\section{Special cases of identity (1).}

COROLlaRY 2. For each complex number $x$ such that $|x|<1$,

$$
\begin{aligned}
& \text { (3) } \prod_{n=1}^{\infty}\left(1-x^{2 n}\right)\left(1+x^{2 n-1}\right)^{4}=\left\{\sum_{n=-\infty}^{\infty} x^{2 m^{2}}\right\}^{2}+x\left\{\sum_{n=-\infty}^{\infty} x^{2 n(n+1)}\right\}^{2} \text {; } \\
& \text { (4) } \prod_{n=1}^{\infty}\left(1-x^{2 n}\right)^{2}\left(1-x^{2 n-1}\right)^{4}=\left\{\sum_{n=-\infty}^{\infty} x^{2 m^{2}}\right\}^{2}-x\left\{\sum_{n=-\infty}^{\infty} x^{2 n(n+1)}\right\}^{2} \text {. }
\end{aligned}
$$

Proof. To obtain (3) let $a=1$ in (1); and, to obtain (4) let $a=-1$.

COROLLARY 3. For each complex number $x$ such that $|x|<1$,

$$
\begin{aligned}
\prod_{n=1}^{\infty}\left(1-x^{n}\right)^{2}= & \sum_{n=-\infty}^{\infty} x^{3 n^{2}} \sum_{n=-\infty}^{\infty} x^{n(3 n+1)} \\
& -x \sum_{n=-\infty}^{\infty} x^{3 n(n+1)} \sum_{n=-\infty}^{\infty} x^{n(3 n+2)} .
\end{aligned}
$$

PROof. For $x=0$ the identity is trivially valid. For $x \neq 0$, in (1) we replace $a$ and $x$ by $-x^{1 / 2}$ and $x^{3 / 2}$, respectively.

COROLlARY 4. For each complex number $x$ such that $|x|<1$,

$$
\begin{aligned}
\prod_{n=1}^{\infty}\left(1-x^{n}\right)^{6}= & \sum_{n=-\infty}^{\infty} x^{n^{2}} \sum_{n=0}^{\infty}(2 n+1)^{2} x^{n(n+1)} \\
& -\sum_{n=-\infty}^{\infty} x^{n(n+1)} \sum_{n=1}^{\infty}(2 n)^{2} x^{n^{2}}
\end{aligned}
$$

Proof. For $x=0$ the identity is trivially valid. For $x \neq 0$ let $a=-x e^{2 i t}$, so that (1) becomes

$$
\begin{aligned}
\left(1-e^{-2 i t}\right)^{2} \prod_{n=1}^{\infty} & \left(1-x^{2 n}\right)^{2}\left(1-2 x^{2 n} \cos 2 t+x^{4 n}\right)^{2} \\
= & \sum_{n=-\infty}^{\infty} x^{2 n^{2}} \sum_{n=-\infty}^{\infty} x^{2 n(n+1)} e^{4 n i t} \\
& -\sum_{n=-\infty}^{\infty} x^{2 n(n+1)}\left\{\sum_{n=1}^{\infty} x^{2 n^{2}} e^{(4 n-2) i t}+\sum_{n=0}^{\infty} x^{2 n^{2}} e^{-(4 n+2) i t}\right\} .
\end{aligned}
$$

For brevity put $f(t)=\Pi\left(1-x^{2 n}\right)^{2}\left(1-2 x^{2 n} \cos 2 t+x^{4 n}\right)^{2}, c(x)=\sum x^{2 n^{2}}, d(x)=$ $\sum x^{2 n(n+1)}$ and multiply both sides of the foregoing identity by $-4^{-1} e^{2 i t}$ to get

$$
\left(\sin ^{2} t\right) f(t)=-\frac{1}{2} c(x) \sum_{n=0}^{\infty} x^{2 n(n+1)} \cos (4 n+2) t+\frac{1}{4} d(x) \sum_{n=-\infty}^{\infty} x^{2 n^{2}} \cos 4 n t .
$$

Differentiating both sides of (7) twice with respect to $t$, we have

$$
\begin{aligned}
& 2 \cos ^{2} t \cdot f(t)+2 \sin t \cdot D_{t}\{\cos t \cdot f(t)\}+D_{t}\left\{\sin ^{2} t \cdot f^{\prime}(t)\right\} \\
& \quad=2 c(x) \sum_{n=0}^{\infty} x^{2 n(n+1)}(2 n+1)^{2} \cos (4 n+2) t-2 d(x) \sum_{n=1}^{\infty} x^{2 n^{2}}(2 n)^{2} \cos 4 n t .
\end{aligned}
$$


Now, setting $t=0$, cancelling a factor of 2 from both sides of the resulting identity and realizing that $f(0)=\Pi\left(1-x^{2 n}\right)^{6}$, we replace $x$ by $x^{1 / 2}$ to obtain identity (6).

4. A theorem of Ramanujan. The theorem which here concerns us is

THEOREM 5. For each nonnegative integer $m$,

$$
p(7 m+5) \equiv 0 \quad(\bmod 7) .
$$

[For a given positive integer $n, p(n)$ denotes the number of unrestricted partitions of $n$; and, conventionally, $p(0)=1$.]

In his original proof $[1$, p. 88$]$ Ramanujan begins by squaring Jacobi's triangular-number identity:

$$
\prod_{n=1}^{\infty}\left(1-x^{n}\right)^{3}=\sum_{n=0}^{\infty}(-1)^{n}(2 n+1) x^{n(n+1) / 2} .
$$

We here replace the square of Jacobi's identity by identity (6), and then multiply by $x^{2}$ to get

$$
x^{2} \prod_{n=1}^{\infty}\left(1-x^{n}\right)^{6}=\sum_{i=-\infty}^{\infty} \sum_{j=0}^{\infty}(2 j+1)^{2} x^{k}-\sum_{r=-\infty}^{\infty} \sum_{s=1}^{\infty}(2 s)^{2} x^{0},
$$

where $k=k(i, j)=i^{2}+j(j+1)+2 ; v=v(r, s)=r(r+1)+s^{2}+2$. We now seek necessary conditions for divisibility of $k$ and $v$ by 7 . Since $k$ and $v$ are symmetrically constructed in terms of squares and triangular numbers, it suffices to deal with $k$. Now,

$$
(2 i)^{2}+(2 j+1)^{2}+7=4 k
$$

Hence, $k \equiv 0(\bmod 7)$ implies

$$
(2 i)^{2}+(2 j+1)^{2} \equiv 0(\bmod 7) .
$$

And, the foregoing congruence is satisfied only if $2 i \equiv 0$ and $2 j+1 \equiv 0(\bmod 7)$. Thus, $k$ and $v$ can be divisible by 7 only if $2 j+1$ and $2 s$, respectively, are divisible by 7 , and consequently the coefficient of $x^{7 m+7}$ in $x^{2} \Pi_{n=1}^{\infty}\left(1-x^{n}\right)^{6}$ is divisible by 7 .

The proof is now completed just as in [1, p. 88].

\section{REFERENCES}

1. G. H. Hardy, Ramanujan, Chelsea, New York, 1959.

2. G. H. Hardy and E. M. Wright, An introduction to the theory of numbers, 4th ed., Clarendon Press, Oxford, 1960.

3. P. A. MacMahon, Combinatory analysis. Vol. 2, Chelsea, New York, 1960.

Department of Mathematical Sciences, Northern Illinois University, DeKalb, Illinois 60115 